\title{
Understanding the alkali - Germanium interaction in the ternary Zintl phase Li18Na2Ge17: First-principles study
}

\author{
Souraya Goumri-Said* \\ Department of physics, College of Science, Alfaisal University, Saudi Arabia
}

\begin{abstract}
The Zintl ternary phase, $\mathrm{Li}_{18} \mathrm{Na}_{2} \mathrm{Ge}_{17}$, have been synthesized typically from a very electropositive metals $(\mathrm{Ge})$ and more electronegative main group metals $\mathrm{Na}$ and $\mathrm{Li}$. We attempted to understand its electronic structure and the contribution of electropositive and electronegative groups by employing first-principles calculations. $\mathrm{Li}_{18} \mathrm{Na}_{2} \mathrm{Ge}_{17}$ is a semiconductor with a narrower and indirect band gap about $0.254 \mathrm{eV}$. Charge density difference distribution supplemented by Bader population were reported to characterize the bonding. As a ternary Zintl, $\mathrm{Li}_{18} \mathrm{Na}_{2} \mathrm{Ge}_{17}$ is obeying to the Zintl-Klemm concept, where an electron transfer from the alkali metal atoms $(\mathrm{Li}, \mathrm{Na})$ to the clusters $\left(\mathrm{Ge}_{17}\right)$ is demonstrated.
\end{abstract}

\section{Introduction}

Zintl clusters have been receiving extensive and lasting interest because of their interesting and diversified structures as well as chemical bonding, unique reactivity, and applications in materials science [1-4]. In fact, Zintl compounds embodying isolated clusters of more than four atoms have been discovered in the early of eighties [3]. These compounds have demonstrated to obey to the Zintl-Klemm concept, known to be "the most important theoretical concept in solidstate chemistry of the last century", where their structural and bonding characteristics depends on the combination of the electropositive metals (e.g. alkali, alkaline earth and rare earth metals) with the maingroup elements (clusters).

The recently synthesized ternary Zintl phase $\mathrm{Li}_{18} \mathrm{Na}_{2} \mathrm{Ge}_{17}$ [5] has been stabilized by mixing cations of different size and different charge, where a novel structure was obtained with favorable cluster sheathing. The single-crystal X-ray structure has revealed that the ternary Zintl phase $\mathrm{Li}_{18} \mathrm{Na}_{2} \mathrm{Ge}_{17}$ contains the anionic cluster unit of $\left[\mathrm{Ge}_{12}\right]^{12-}$ which give to this phase specific features in bonding and electronic properties. In fact, $\mathrm{Li}_{18} \mathrm{Na}_{2} \mathrm{Ge}_{17}$ have various anions of different sizes, mainly $\mathrm{Ge}_{12}$, $\mathrm{Ge}_{4}$ which need a cation (like $\mathrm{Na}_{2}$ or $\mathrm{Li}_{4}$ ) to ensure the cohesion of the compound. Cations are important to stabilize these anionic clusters where they can play a role of bridge or separator [6]. The aim of the present paper is to provide a comprehensive picture of the bonding properties and electronic structure of the ternary $\mathrm{Li}_{18} \mathrm{Na}_{2} \mathrm{Ge}_{17}$. In particular, we addressed the crystal structure, related electronic properties as well as bonding analysis by using ab-initio calculations based on the density functional theory. The electronic structure and chemical bonding investigation have then provided an overall view of electro-structural behavior that can be needed for improving its applications.

\section{Computational details}

Our calculations were performed using the Quantum Wise (ATK) package [7] with the generalized gradient approximation (GGA) of Perdew, Burke and Ernzerhof [8]. ATK code is one of the electronic structure-modeling method as well as software package developed within density functional theory (DFT) by making a use of standard norm-conserving pseudopotential (PP) besides the application of flexible linear numeric combination of atomic orbitals basis set [9]. In these calculations numerical double- $\zeta$ plus polarization (DZP) basis set were used [10]. In order to avoid any underestimation in the band gap energy, we used the modified version of the exchange potential proposed by Becke and Johnson (mBJ or meta-GGA) exchange correlation functional [11] and the improved Troullier-Martins normconserving pseudopotentials were adopted for all atoms in the Zintl. The structural geometry were optimized by reducing the atomic forces of atoms to be smaller than $0.05 \mathrm{eV} / \mathrm{A}$. The real space grid for the electrostatic potentials is calculated with the mesh cut-off energy of 450 $\mathrm{eV}$ which realizes the balance between efficiency and accuracy in the calculation. For Brillouin zone (BZ) sampling, grid of $2 \times 2 \times 4$ k-points for relaxation and structural optimization, and grid of $4 \times 4 \times 8 \mathrm{k}$-points for density of states calculation were used. The choice of number of $k$ points and the value of plane wave cutoff energy were considered to ensure the convergence criteria of total energy.

\section{Results and discussion}

The $\mathrm{Li}_{18} \mathrm{Na}_{2} \mathrm{Ge}_{17}$ crystallizes in the trigonal space group P31m (No. 157) where the unit cell parameters were found to be $\mathrm{a}=17.0905(4)$ $\AA$, and $c=8.0783(2) \AA$, with $\alpha=\beta=90$ and $\gamma=120$ [6] (see Figure1a). Note that the structure contains three different $Z$ intl anions $(Z=3)$. The $\mathrm{Li}_{18} \mathrm{Na}_{2} \mathrm{Ge}_{17}$ is a ternary of a series of Zintl phases, issued from three different Zintl anions: isolated anions $\mathrm{Ge}^{4-}$, tetrahedra $\left[\mathrm{Ge}_{4}\right]^{4-}$, and truncated, Li-centered tetrahedra $\left[\mathrm{Li} @ \mathrm{Ge}_{12}\right]^{11-}$, whose hexagonal faces are capped by four Li cations, resulting in the polyhedra $\left[\mathrm{Li}_{\mathrm{Li}} \mathrm{Li}_{42}\right]^{7-}$ as displayed in Figure 1(b-d). Moreover, the structure incorporates

Correspondence to: Souraya Goumri-Said, Department of physics, College of Science, Alfaisal University, P.O. Box 50927, Riyadh 11533, Saudi Arabia, E-mail: sosaid@alfaisal.edu

Key words: Zintl phases, Zintl-Klemm concept, DFT, Bader analysis

Received: January 04, 2018; Accepted: January 28, 2018; Published: January 31, 2018 
other coordination environments as the isolated Ge atom Gel is coordinated by nine $\mathrm{Li}$ atoms and $\mathrm{Na} 1$ (Figure $1 \mathrm{e}$ ). The $\mathrm{Li}_{18} \mathrm{Na}_{2} \mathrm{Ge}_{17}$ structure was relaxed at the experimental lattice parameters. Lattice relaxation was studied, but the atomic positions stayed very close to the positions dictated by symmetry and the lattice constant varied by only about $1 \%$, as reported in Table 1 . As can be seen from these data, the relaxed $\mathrm{Ge} 4-\mathrm{Ge} 5$ and $\mathrm{Ge} 2-\mathrm{Ge} 3$ distances within the tetrahedra $\left[\mathrm{Ge}_{4}\right]^{4-}$, are $2.599 \AA$ and $2.604 \AA$, respectively. These results are agree well with the experimental values of $2.569 \AA$ and $2.575 \AA$. Moreover, the optimized equilibrium parameters, $a=17.186 \AA$, and $c=8.106 \AA$, were found to corroborate with the experimental data [10]. The deviations from experimental values were overestimated for all quantities by $0.56 \%$ and $0.34 \%$, respectively.

To understand the interplay between the electronic and crystal structures, theoretical analysis of the band structure and total/ partial density of states (DOS) has been carried out for $\mathrm{Li}_{18} \mathrm{Na}_{2} \mathrm{Ge}_{17}$ with complete occupation of all positions. As shown in Figure 2 (a, $\mathrm{b})$, the band structure and DOS reveal an indirect narrow band gap $(\mathrm{A} \rightarrow \Gamma)$ approximately $0.254 \mathrm{eV}$ above the Fermi level and indicate semiconducting behavior. The partial DOS below the Fermi level consists three well separated regions. The low-energy range $(\mathrm{E}<-6.5$ $\mathrm{eV}$ ) is composed mainly of the $\mathrm{Ge}(\mathrm{s})$ states with minor participation of $\mathrm{Li}(\mathrm{s}), \mathrm{Na}(\mathrm{s}), \mathrm{Na}(\mathrm{p})$, and $\mathrm{Ge}(\mathrm{p})$ states (see Figure $3(\mathrm{a}, \mathrm{b})$ ). The region between $\sim-4$ and $-0.6 \mathrm{eV}$ is mainly formed by the $\mathrm{Ge}(\mathrm{p})$ states with small contribution of $\mathrm{Li}(\mathrm{s})$, and $\mathrm{Na}(\mathrm{p})$. Furthermore, strong Ge-s orbital contributions to the DOS also appear between -4 and $-0.6 \mathrm{eV}$ below the Fermi level as expected for negatively charged $\mathrm{Ge}$ atoms. The bands from $-0.5 \mathrm{eV}$ to the Fermi level are mainly composed of Ge $4 \mathrm{p}$ and $\mathrm{Na} 3 \mathrm{~s}$ orbitals. Further analysis of the projected DOS clearly reveals a hybridization between $4 \mathrm{p}$ orbitals of Ge with $2 \mathrm{p}$ orbitals of $\mathrm{Na}$ (in the range from $-0.5 \mathrm{eV}$ to the Fermi), while orbitals of Li do not have much mixing with Ge orbitals.

Now we turn to the alkali-metals $\mathrm{Na}$ and $\mathrm{Li}$ contributions as presented in the electronic structure. The alkali-metals cations was playing an important role in linking or separating different Ge clusters and contributed to stabilize the Zintl anions [6]. In $\mathrm{Li}_{18} \mathrm{Na}_{2} \mathrm{Ge}_{17}$ structure, the $\mathrm{Li}$ atoms occupy two sites: the first position is located in the center

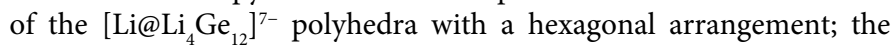
second position is situated outside of the polyhedra. However, we have plotted the PDOS Li s states of each position in Figure 3b. It can be stated that the Li $2 \mathrm{~s}$ states related to $\left[\mathrm{Li} @ \mathrm{Li}_{4} \mathrm{Ge}_{12}\right]^{7-}$ polyhedra show a complete electron transfer from the Li (Alkali atom) to the cluster $\left(\mathrm{Ge}_{12}\right)$ compared to the Li located outside the cluster. This result shows that $\mathrm{Li}_{18} \mathrm{Na}_{2} \mathrm{Ge}_{17}$ fulfills the concept of Zintl-Klemm [12, 13]. Further insight into the organization of the crystal structures was obtained by applying the Bader analysis of atomic interactions [13]. Bader charge
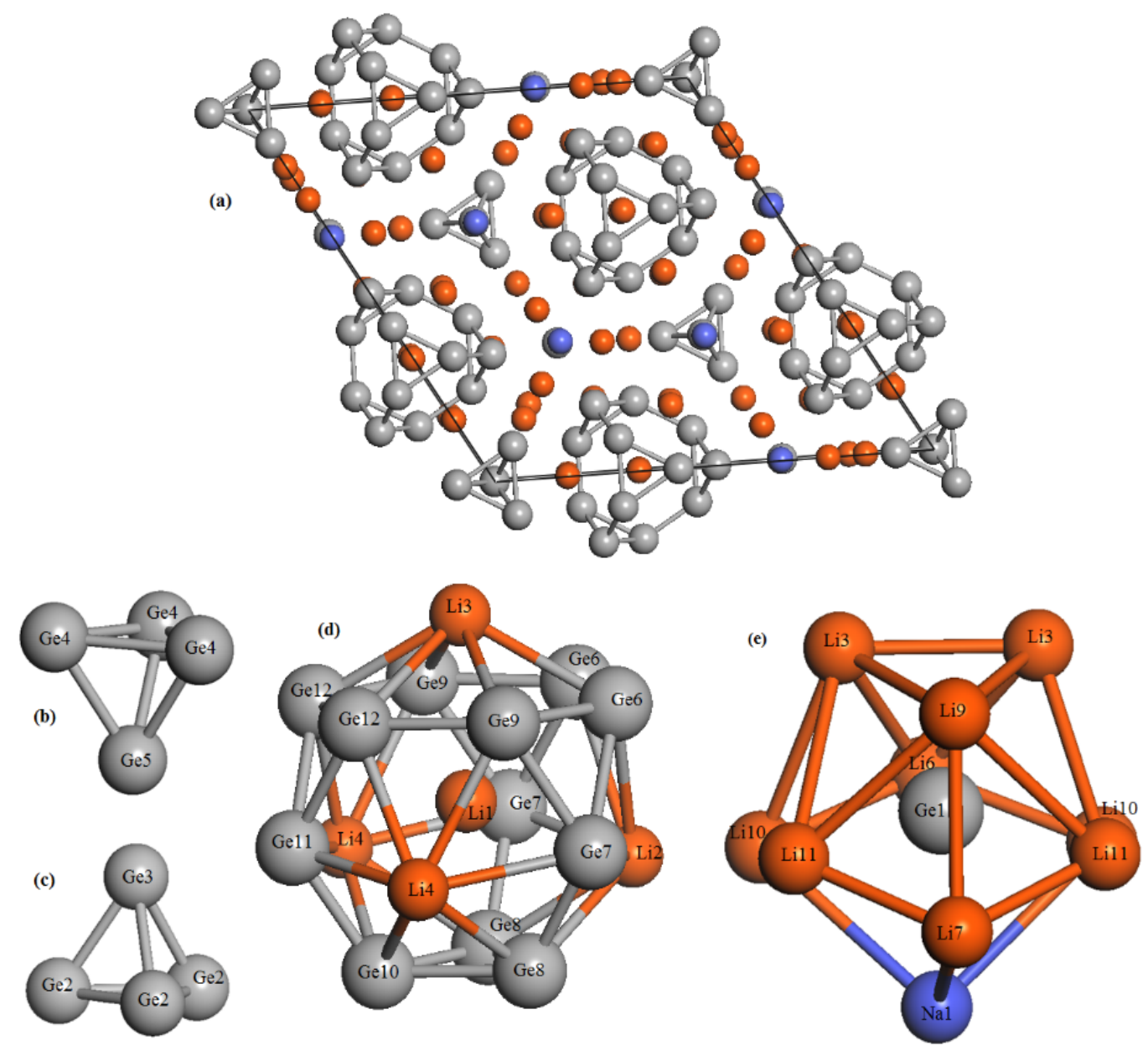

Figure 1. (a) Crystal structure of $\mathrm{Li}_{18} \mathrm{Na}_{2} \mathrm{Ge}_{17}$ ( $\mathrm{Li}$, orange; $\mathrm{Na}$, bleu; $\mathrm{Ge}$, gray), (b, c) different tetrahedra $[\mathrm{Ge} 4]^{4-}$,

(d) Structure of the Li-centered Friauf polyhedron $\left[\mathrm{Li} @ \mathrm{Li}_{4} \mathrm{Ge}_{12}\right]^{7-}$, and

(e) the isolated $\mathrm{Ge}$ atom $\mathrm{Ge} 1$ is coordinated by nine $\mathrm{Li}$ atoms and $\mathrm{Na1}$ ( $\mathrm{Li}$, orange; $\mathrm{Na}$, bleu; Ge, gray) 



Figure 2. (a) Electronic band structure and (b) total density of states of Li18Na2Ge17.

The vertical solid line denotes the Fermi level.
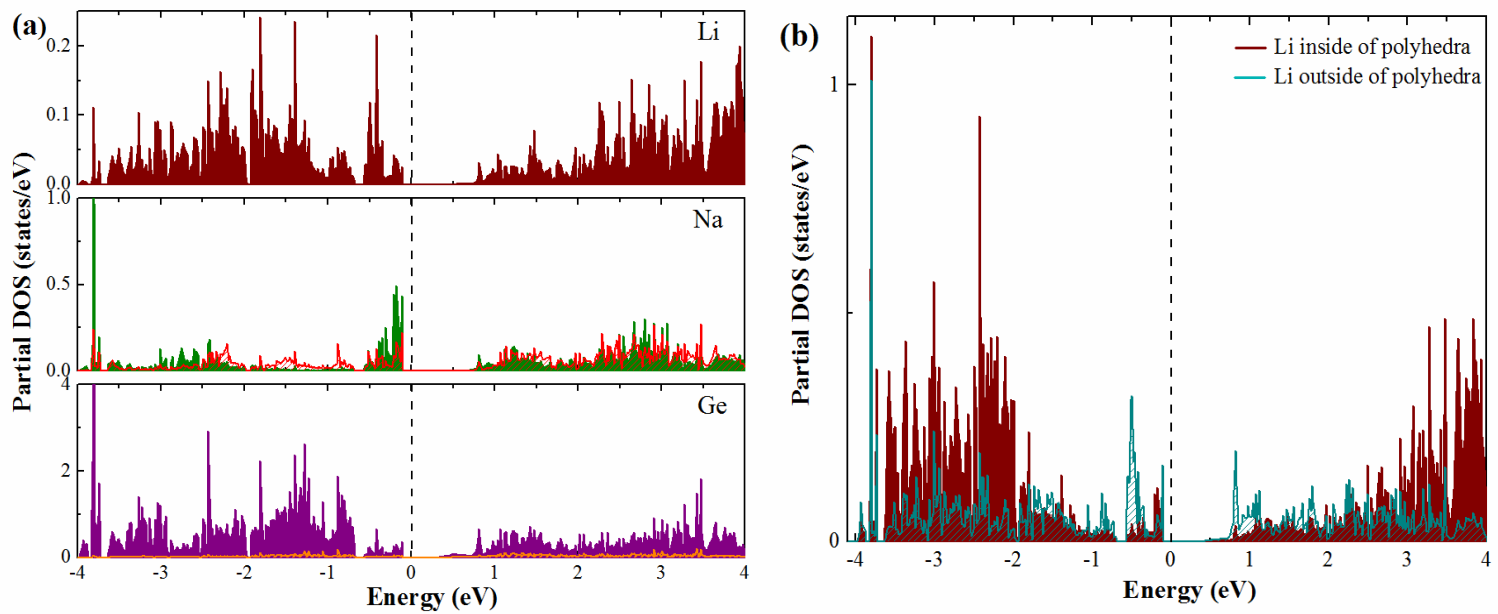

Figure 3. (a) Calculated partial density of states plots for $\mathrm{Li}_{18} \mathrm{Na}_{2} \mathrm{Ge}_{17}$ compound. Wine, green, red, orange and purple areas represent PDOS of the Li $s$ states, Na $\mathrm{s}, \mathrm{p}$ states and Ge $\mathrm{s}$, $\mathrm{p}$ states, respectively.

(b) Partial projections of orbital components Li 2s inside (wine area) and outside (dark cyan area) of polyhedron $\left[\mathrm{Li}_{0} @ \mathrm{Li}_{4} \mathrm{Ge}_{12}\right]^{7-}$.

Table 1. Selection of distances of new Zintl phase $\mathrm{Li}_{18} \mathrm{Na}_{2} \mathrm{Ge}_{17}$.

\begin{tabular}{|c|c|c|c|c|c|}
\hline & Our work & Exp. & & Our work & Exp. \\
\hline Ge1 - Li6 & 2.539 & 2.514 & $\mathrm{Ge} 7-\mathrm{Li} 2$ & 3.007 & 2.980 \\
\hline $\mathrm{Ge} 1-\mathrm{Li} 7$ & 2.742 & 2.709 & $\mathrm{Ge} 7-\mathrm{Li} 4$ & 2.882 & 2.882 \\
\hline Ge1 - Li9 & 2.592 & 2.560 & $\mathrm{Ge} 8-\mathrm{Ge} 8$ & 2.734 & 2.695 \\
\hline Ge1 - Li11 & 2.569 & 2.543 & Ge $8-\mathrm{Ge} 10$ & 2.592 & 2.560 \\
\hline Ge1 - Li13 & 2.671 & 2.643 & $\mathrm{Ge} 8-\mathrm{Li} 2$ & 2.926 & 2.916 \\
\hline Ge1-Li10 & 2.927 & 2.902 & $\mathrm{Ge} 8-\mathrm{Li} 4$ & 2.842 & 2.851 \\
\hline $\mathrm{Ge} 2-\mathrm{Ge} 3$ & 2.604 & 2.575 & $\mathrm{Ge} 9-\mathrm{Ge} 12$ & 2.522 & 2.500 \\
\hline $\mathrm{Ge} 2-\mathrm{Ge} 2$ & 2.598 & 2.561 & $\mathrm{Ge} 9-\mathrm{Li} 3$ & 2.944 & 2.935 \\
\hline $\mathrm{Ge} 4-\mathrm{Ge} 5$ & 2.599 & 2.569 & $\mathrm{Ge} 9-\mathrm{Li} 4$ & 3.008 & 2.978 \\
\hline $\mathrm{Ge} 4-\mathrm{Ge} 4$ & 2.627 & 2.582 & Ge10-Ge11 & 2.504 & 2.484 \\
\hline Ge6 - Ge6 & 2.516 & 2.488 & Ge11-Ge12 & 2.619 & 2.592 \\
\hline $\mathrm{Ge} 6-\mathrm{Ge} 7$ & 2.698 & 2.657 & Ge11 - Li4 & 2.870 & 2.871 \\
\hline Ge6-Ge9 & 2.629 & 2.598 & $\mathrm{Ge} 12-\mathrm{Ge} 12$ & 2.609 & 2.599 \\
\hline Ge6 - Li2 & 3.001 & 2.974 & Ge12 - Li3 & 2.881 & 2.857 \\
\hline Ge6 - Li3 & 2.921 & 2.906 & Li1 - Li3 & 2.992 & 2.981 \\
\hline $\mathrm{Ge} 7-\mathrm{Ge} 8$ & 2.532 & 2.495 & $\mathrm{Li} 1$ - Li2 & 2.936 & 2.940 \\
\hline $\mathrm{Ge} 7-\mathrm{Ge} 9$ & 2.596 & 2.565 & Li1 - Li4 & 2.992 & 3.009 \\
\hline
\end{tabular}




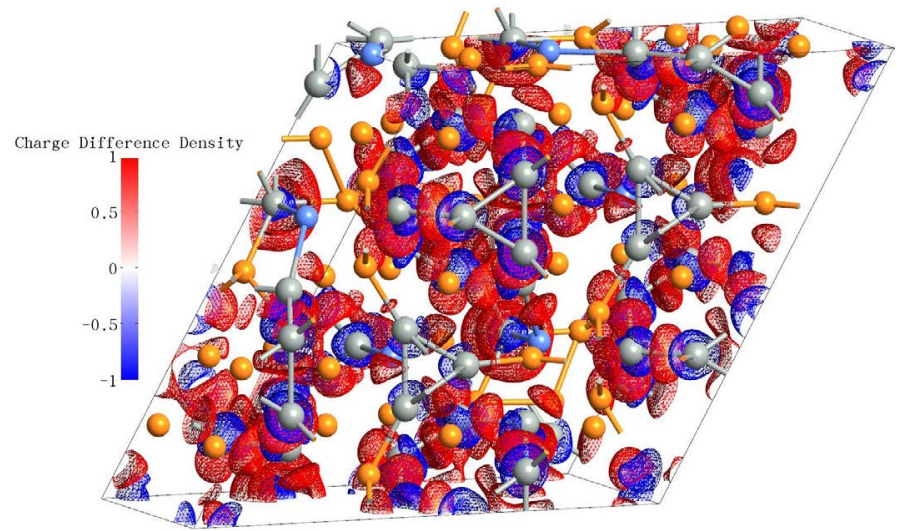

Figure 4. Charge density isosurfaces displaying the bonding character of $\mathrm{Li}_{18} \mathrm{Na}_{2} \mathrm{Ge}_{17}$.

analysis indicates that the isolated $\mathrm{Ge}$ atom, coordinated by nine $\mathrm{Li}$ atoms and $\mathrm{Na}$ (Figure 1e), has acquired more the charges about -2.964 than the Ge tetrahedron -1.198e (Figure 1b,c), and polyhedral -0.841e (Figure 1d). In accordance with the electronegativities of the constituting elements, the Ge species have the largest average negative charge $-1.28 \mathrm{e}$, while the $\mathrm{Li}$ and $\mathrm{Na}$ show a positive charge to be $+0.886 \mathrm{e}$ and +0.826 e, respectively. Obviously, the charge transfer is playing an important role in the organization of the $\mathrm{Li}_{18} \mathrm{Na}_{2} \mathrm{Ge}_{17}$ crystal structure.

Analysis of the charge density difference reveals maxima of the charge accumulation between Ge atoms and shows a charge especially around the Li-centered Friauf polyhedron $\left[\mathrm{Li} @ \mathrm{Li}_{4} \mathrm{Ge}_{12}\right]^{7-}$ framework, as shown in figure 4. Thus, $\mathrm{Ge}^{12-}$ is completed to $\left[\mathrm{Li}_{4} \mathrm{Li}_{4} \mathrm{Ge}_{12}\right]^{7-}$ deltahedral cluster of 17 atoms with covalent bonds [14]. Furthermore, the charge density isosurface is showing that the occupied bonding and nonbonding states are always centered at Ge atoms. These calculations confirm the Bader population analysis and clearly show that the charge is localized in both, the bonding and the lone pair regions [15].

In summary, we used the state of art of density functional theory to analyze the electronic structure and bonding characteristics of the ternary Zintl phase $\mathrm{Li}_{18} \mathrm{Na}_{2} \mathrm{Ge}_{17}$. Our calculations have revealed the existence of a balance between packing efficiency and structure, electronic nature, and ions interactions.

\section{Acknowledgments}

Financial support for this study is acknowledged in the form of an internal grants: IRG 18418 from office of research at Alfaisal University.

\section{References}

1. Corbett JD (2000) Polyanionic Clusters and Networks of the Early p-Element Metals in the Solid State: Beyond the Zintl Boundary Angew Chem Int. Ed. 39: 670-690.

2. Arnold M. Guloy (1996) Chemistry, Structures and Bonding of Zintl Phases and Ions; Kauzlarich, S. M., Ed. VCH: Weinheim, Germany p: 245.

3. Von Schnering HG (1981) Angew. Chem. Int. Ed. Engl. 93: 20-33,44.

4. Scharfe S, Kraus F, Stegmaier S, Schier A, Fässler TF (2011) Zintl ions, cage compounds, and intermetalloid clusters of Group 14 and Group 15 elements. Angew Chem Int Ed Engl 50: 3630-3670.

5. Sevov SC, Goicoechea JM (2006) Chemistry of Deltahedral Zintl Ions. Organometallics 25: $5678-5692$.

6. Scherf LM, Zeilinger M, Fässler TF (2014) Li18Na2Ge17-A Compound Demonstrating Cation Effects on Cluster Shapes and Crystal Packing in Ternary Zintl Phases. Inorg Chem 53: 2096-2101.

7. Soler JM, Artacho E, Gale JD, Garcia A, Junquera J, et al. (2002) The SIESTA method for ab initio order-N materials simulation. D JPhys Condens Matter 14: 2745-2779.

8. Perdew JP, Burke K, Ernzerhof M (1996) Generalized Gradient Approximation Made Simple Phys Rev Lett 77: 3865.

9. Troullier N, Martins JL (1991) Efficient pseudopotentials for plane-wave calculations. Phys Rev B 43: 1993.

10. Junquera J, Paz Ó, Sánchez-Portal D, Artacho E (2001) Numerical atomic orbitals for linear-scaling calculations Phys Rev B 64: 235111.

11. Tran F, Blaha P (2009) Accurate Band Gaps of Semiconductors and Insulators with a Semilocal Exchange-Correlation Potential Phys Rev Lett 102: 226401.

12. Bobev S, Sevov SC (2001) Synthesis and Characterization of RbLi7Ge8 with Isolated closo-[Li4Ge12]8- Ions, Lithium-Capped Truncated Tetrahedra of Ge1212-. Angew Chem Int Ed. 40: 8 .

13. Hoser AA, Jarzembska KN, Dobrzycki Ł, Gutmann MJ, Woźniak K (2012) Differences in Charge Density Distribution and Stability of Two Polymorphs of Benzidine Dihydrochloride. Cryst. Growth Des 12: 3526-3539.

14. Bader RFW (1994) Atoms in Molecules, A Quantum Theory; Clarendon Press and Oxford University Press Inc: New York.

15. Nesper R (2003) Structural and Electronic Systematics in Zintl Phases of the Tetrels. In: Jutzi P, Schubert U (eds). Silicon Chemistry. Wiley-VCH, Weinheim, 171.

Copyright: (C2018 Goumri-Said S. This is an open-access article distributed under the terms of the Creative Commons Attribution License, which permits unrestricted use, distribution, and reproduction in any medium, provided the original author and source are credited. 